\title{
The Hölder and the Besov regularity of the density for the solution of a parabolic stochastic partial differential equation
}

\author{
PIERRE-LUC MORIEN
}

Laboratoire de Probabilités (Unité de Recherche associée au CNRS 224), Université Paris 6, 4 place Jussieu, 75252 Paris, France.e-mail: morien@modalx.u-paris10.fr

In this paper we prove that the density $p_{t, x}(y)$ of the solution of a white-noise-driven parabolic stochastic partial differential equation (SPDE) satisfying a strong ellipticity condition is $\frac{1}{2}$ Lipschitz continuous with respect to (w.r.t.) $t$ and $1-\epsilon$ Lipschitz continuous w.r.t. $x$ for all $\epsilon \in] 0$, 1 [. In addition, we show that it belongs to the Besov space $B_{1, \infty, \infty}$ w.r.t. $x$. The proof is based on the Malliavin calculus of variations and on some refined estimates for the Green kernel associated with the SPDE.

Keywords: Besov spaces; Malliavin calculus; parabolic SPDEs

\section{Introduction}

Consider the following parabolic stochastic partial differential equation:

$$
\frac{\partial X}{\partial t}(t, x)=\frac{\partial^{2} X}{\partial x^{2}}(t, x)+\psi(X(t, x))+\varphi(X(t, x)) \dot{W}(t, x),
$$

for $(t, x) \in] 0, T[\times] 0,1[$, where $W$ is a space-time white noise on $[0, T] \times[0,1], \varphi$ and $\psi$ some smooth functions with bounded derivatives, satisfying the Neumann boundary conditions:

$$
X(0, x)=X_{0}(x), \quad \frac{\partial X}{\partial x}(t, 0)=\frac{\partial X}{\partial x}(t, 1)=0 .
$$

The solution of such an equation is given by the following evolution equation (Walsh 1986): $X(t, x)=G_{t}\left(x, X_{0}\right)+\int_{0}^{t} \int_{0}^{1} G_{t-s}(x, y) \varphi(X(s, y)) W(\mathrm{~d} y, \mathrm{~d} s)+\int_{0}^{t} \int_{0}^{1} G_{t-s}(x, y) \psi(X(s, y)) \mathrm{d} y \mathrm{~d} s$,

where $G_{t}$ denotes the Green kernel related to the heat equation on $[0, T] \times[0,1]$ with the Neumann boundary conditions and where we set $G_{t}(x, \phi)=\int_{0}^{1} G_{t}(x, y) \phi(y) \mathrm{d} y$ for any continuous function $\phi$ on $[0,1]$. 
Bally and Pardoux (1994) have established that, for each fixed $(t, x) \in] 0, T[\times[0,1]$, $X(t, x)$ is an infinitely differentiable functional in the sense of the Malliavin calculus related to $W$ and that, on the set $\{\varphi \neq 0\}, X(t, x)$ possesses a $C^{\infty}$ density $y \mapsto p_{t, x}(y)$ with respect to (w.r.t.) the Lebesgue measure.

The aim of this paper is to study the regularity of the function $(t, x) \mapsto p_{t, x}(y)$ under the strong ellipticity condition $\varphi \geqslant c>0$. A natural direction to investigate is that of the Hölder regularity. Here we prove that the density function is $\frac{1}{2}$ Lipschitz w.r.t. $t$ and $1-\epsilon$ Lipschitz w.r.t. $x$.

The first thing to note is that the regularity that we claim is stronger than the pathwise regularity of $X(t, x)$, since $X(t, x)$ is $\frac{1}{4}-\epsilon$ Lipschitz w.r.t. $t$ and $\frac{1}{2}-\epsilon$ Lipschitz w.r.t. $x$ (see, for example, Walsh (1986)). However, this apparent "increase" in regularity is not surprising. Indeed, consider the standard Brownian motion $B_{t}$ on $\mathbb{R}$. It is well known that $B_{t}$ is $\frac{1}{2}-\epsilon$ Lipschitz w.r.t. $t$, whereas its density is differentiable. A heuristic way to understand what yields this "new" regularity is to note that the density $p_{t}(y)$ can be seen as the limit of $\mathbb{E}\left[f\left(B_{t}\right)\right]$ when $f \rightarrow \delta_{y}$. Then, using the Itô formula, one observes that the stochastic integrals involved, which account for most of the loss of regularity, are "killed" by the expectation.

This particular example gives two important ideas. First, a convenient way to obtain the required Hölder regularity for the density function $p_{t, x}$ w.r.t. its parameters is to show estimates of the following kind:

$$
\mid \mathbb{E}\left[f(X(t+h, x)]-\mathbb{E}\left[f(X(t, x)] \mid \leqslant C\left\|F_{f}\right\|_{\infty} h^{1 / 2}\right.\right.
$$

and

$$
\mid \mathbb{E}\left[f(X(t, x+h)]-\mathbb{E}\left[f(X(t, x)] \mid \leqslant C^{\prime}\left\|F_{f}\right\|_{\infty} h^{1-\epsilon},\right.\right.
$$

where $F_{f}$ denotes an antiderivative of $f$, and where $f$ is a function with sufficient regularity.

Then it only remains to take a sequence $f_{n, y}$ of functions converging towards the Dirac measure $\delta_{y}$, so that $\left\|F_{n, y}\right\|_{\infty}$ is uniformly bounded w.r.t. $n$ and $y$ (e.g. Gaussian kernels).

The second idea given by the example above is to take advantage of the effect of the expectation on stochastic integrals (an effect which is lost when using such inequalities as the Burkholder inequality). However, it is impossible to use the Itô formula in our context, for the representation of $X(t, x)$ given by the evolution equation (1.1) is not a semimartingale decomposition. A natural idea is then to employ a Taylor expansion of order 2. One then has to find a way to deal with such quantities as $\mathbb{E}\left[f^{\prime}(X(t, x)) U_{t, x, h}\right]$, where $U$ is a stochastic integral of the Itô type. In a standard context, one would apply the Itô formula to $f^{\prime}(X(t, x))$. Here we use instead the crucial fact that, for adapted processes, the Itô integral corresponds to the Skorohod integral, which enables us to employ the standard duality formula between the derivative operator (in the Malliavin sense) and the Skorohod integral, thus producing classical integrals that are more manageable.

Yet technical difficulties arise because of the presence of the Green kernel $G$, which has a singularity at $t=0$. Therefore, new estimates have to be found for $G$ (the inequalities proved by Walsh and quoted by Bally et al. (1995) will also be quite useful).

Finally we note the discrepancy between time and space regularity and wonder whether the result concerning the latter may be improved, and in which sense. A natural approach is 
to consider the following functional vector space: let $f$ be a real function defined on $[0,1]$, we define, for $h \in[0,1]$ and $x \in[0,1]$ such that $[x-h, x+h] \subset[0,1]$, the following (iterated) differences:

$$
\Delta_{h}(f)(x)=f(x+h)-f(x), \Delta_{h}^{2}(f)(x)=\Delta_{h}\left(\Delta_{h}\right)(f)(x)=f(x+h)+f(x-h)-2 f(x) .
$$

Then one defines the norm $\|\cdot\|_{1, \infty, \infty}$ by

$$
\|f\|_{1, \infty, \infty}:=\|f\|_{\infty}+\sup _{|h| \leqslant 1} \frac{\left\|\Delta_{h}^{2}(f)\right\|_{\infty}}{|h|} .
$$

The space of real-valued functions $f$ such that $\|f\|_{1, \infty, \infty}<\infty$ is denoted by $B_{1, \infty, \infty}$ and is a Besov space. We remark that the space $B_{1, \infty, \infty}$ (which is sometimes called the Zygmund class) strictly contains the space of 1-Lipschitz-continuous functions. (We refer for instance to the book by Bergh and Löfström (1986) for a more detailed account on the subject.)

We then prove that, for each $\tau \in] 0, T\left[\right.$, the function $x \mapsto p_{t, x}(y)$ belongs to the space $B_{1, \infty, \infty}$ uniformly in $(t, y) \in[\tau, T] \times \mathbb{R}$, i.e. there exists some real constant $C_{\tau}$ such that, for all $(t, y) \in[\tau, T] \times \mathbb{R}$,

$$
\left\|p_{t, \cdot}(y)\right\|_{1, \infty, \infty} \leqslant C_{\tau}<\infty .
$$

The method that we use is a simple refinement of that employed for the Hölder regularity, via new estimates of the Green kernel $G$.

Our work is therefore organized as follows. In Section 2 we expose the context of our study and state our main theorem. The next short section, Section 3, is then devoted to the exposition of the Malliavin calculus related to the white noise $W$.

The proof of the main result (Theorem 2.1) is then given in Section 4. A crucial tool of this proof is a very general result (Proposition 4.2) which roughly states that, if two random variables $F$ and $G$ are close in the sense of some Sobolev norm w.r.t. the Malliavin calculus, then the random variables obtained via the Malliavin integration-by-parts formula are close as well. This result, used via its corollary (Corollary 4.2), will be crucial to give estimates in terms of the norm $\left\|F_{f}\right\|_{\infty}$ for the terms involving $f$ and its derivatives.

Once this result is proved, we first deal with time regularity and then with space regularity. For the latter, we first concentrate on the Hölder regularity and then on the Besov regularity. Some technical lemmas are finally given in Appendix 1.

\section{General framework and statement of the results}

Let $(\Omega, \mathscr{F}, \mathbb{P})$ be a probability space and $W$ a space-time white noise on $[0, T] \times[0,1]$ with covariance $\mathrm{d} s \mathrm{~d} y$, where $T$ is a fixed deterministic time. Let $\mathscr{F}_{t}=$ $\sigma(W(A), A \in \mathscr{B}([0, t] \times[0,1])) \vee \mathscr{N}$ where $\mathscr{N}$ is the class of $\mathbb{P}$-null sets of $\mathscr{F}$ and let $\mathscr{P}$ denote the $\sigma$-algebra of $\mathscr{F}_{t}$-progressively-measurable subsets of $\Omega \times[0, T]$.

Let $X=(X(t, x))$ be the solution of $(\mathrm{E})$. This means that $X$ satisfies the evolution equation (1.1), where 


$$
G_{t}(x, y)=\frac{1}{(4 \pi t)^{1 / 2}} \sum_{n=-\infty}^{n=+\infty}\left\{\exp \left(\frac{-(y-x-2 n)^{2}}{4 t}\right)+\exp \left(\frac{-(y+x-2 n)^{2}}{4 t}\right)\right\},
$$

for which we have (cf. Friedman (1964)):

$$
\text { (*) } \exists C>0, \forall t \in] 0, T], \forall(x, y) \in[0,1]^{2}, G_{t}(x, y) \leqslant \frac{C}{t^{1 / 2}} \exp \left(-\frac{(x-y)^{2}}{4 t}\right) .
$$

In the sequel, we assume that $X_{0} \in C^{1}([0,1]]$ and that $\varphi$ and $\psi$ satisfy the following hypotheses.

(H1) $\varphi, \psi$ and all their derivatives are bounded.

(H2) $\exists c>0, \forall x \in \mathbb{R}, \varphi(x) \geqslant c$.

We remark that (H2) is a condition of strong ellipticity.

Using the Malliavin stochastic calculus of variations, Bally and Pardoux (1993) have proved that under (H1) and (H2), for all $(t, x) \in] 0, T[\times[0,1]$, the solution $X(t, x)$ of (E) has a density $p_{t, x}(y)$ which is infinitely differentiable with respect to $y \in \mathbb{R}$.

The aim of this paper is to determine the regularity of the function $(t, x) \rightarrow p_{t, x}$, that is, we prove the following result.

Theorem 2.1. Let $\tau \in] 0, T[$ be a fixed time.

(1) There exists a constant $C_{\tau}$ such that, for all $t \in[\tau, T], h>0$ such that $t+h \leqslant T$, $y \in \mathbb{R}$ and $x \in[0,1]$, we have

$$
\left|p_{t+h, x}(y)-p_{t, x}(y)\right| \leqslant C_{\tau} h^{1 / 2} .
$$

(2) Let $\epsilon \in] 0,1$. There exists a constant $C_{\tau, \epsilon}$ such that, for all $t \in[\tau, T], x \in[0,1]$, $h>0$ such that $x+h \leqslant 1$, and $y \in \mathbb{R}$, we have

$$
\left|p_{t, x+h}(y)-p_{t, x}(y)\right| \leqslant C_{\tau, \epsilon} h^{1-\epsilon} .
$$

(3) The function $x \mapsto p_{t, x}(y)$ belongs to the space $B_{1, \infty, \infty}$ uniformly in $(t, y) \in$ $[\tau, T] \times \mathbb{R}$, i.e. there exists some real constant $C_{\tau}$ such that, for all $(t, y) \in[\tau, T] \times \mathbb{R}$,

$$
\left\|p_{t, \cdot}(y)\right\|_{1, \infty, \infty} \leqslant C_{\tau}<\infty .
$$

Remark. Theorem 2.1 remains valid in the case of the Dirichlet boundary conditions if we restrict the space variable to intervals of the type $[\alpha, 1-\alpha] \subset[0,1]$, with $\alpha>0$. The only difference with the Neumann case lies in the use of Lemma A1.2, as seen in the proof of Lemma 4.1.

\section{Malliavin calculus}

In this section, we introduce the notation and recall the main results of the Malliavin calculus related to $W$ (for the proofs and a more comprehensive account on the subject, we refer to Nualart (1995) and to Nualart and Sanz (1985) for the special case that we consider here). 


\subsection{The basics}

We denote by $\mathscr{S}$ the space of smooth functionals, i.e. of real-valued functionals of the form

$$
F=f\left(W\left(h_{1}\right), W\left(h_{2}\right), \ldots, W\left(h_{m}\right)\right),
$$

where $f \in C^{\infty}\left(\mathbb{R}^{m}\right)$ and has polynomial growth, as well as its derivatives, and $h_{1}, h_{2}, \ldots, h_{m}$ is an orthonormal sequence in $L^{2}\left(\Lambda_{T} ; \mathrm{d} t \mathrm{~d} x\right)$, with $\Lambda_{t}:=[0, t] \times[0,1]$, and for $h \in L^{2}\left(\Lambda_{T}\right)$, $W(h):=\int_{0}^{T} \int_{0}^{1} h(s, y) W(\mathrm{~d} y, \mathrm{~d} s)$. For $F \in \mathscr{S}$, one defines the first-order Malliavin derivative to be the $L^{2}\left(\Lambda_{T}\right)$ valued random variable

$$
D_{t, x} F=\sum_{i=1}^{m} \partial_{i} f\left(W\left(h_{1}\right), W\left(h_{2}\right), \ldots, W\left(h_{m}\right)\right) h_{i}(t, x)
$$

Similarly, the derivative of order $k$ of $F$ is the $L^{2}\left(\Lambda_{T}^{k}\right)$ valued random variable

$$
D_{\alpha}^{k} F=\sum_{i_{1}, \ldots, i_{k}=1}^{m} \partial_{i_{1}} \ldots \partial_{i_{k}} f\left(W\left(h_{1}\right), W\left(h_{2}\right), \ldots, W\left(h_{m}\right)\right) h_{i_{1}}\left(\alpha_{1}\right) \ldots h_{i_{k}}\left(\alpha_{k}\right),
$$

where $\alpha=\left(\alpha_{1}, \ldots, \alpha_{k}\right), \alpha_{i}=\left(r_{i}, z_{i}\right) \in \Lambda_{T}, 1 \leqslant i \leqslant k$. Then, for $p \geqslant 1$ and $k \in \mathbb{N}$, the space $\mathbb{D}^{k, p}$ is the closure of $\mathscr{S}$ w.r.t. the seminorm

$$
\|F\|_{k, p}=\left(\left(\mathbb{E}|F|^{p}\right)+\sum_{i=1}^{k}\left(\mathbb{E}\left\|D^{i} F\right\|^{p}\right)\right)^{1 / p}, \quad\left\|D^{i} F\right\|^{2}=\int_{\Lambda_{T}^{i}}\left(D_{\alpha}^{i} F\right)^{2} \mathrm{~d} \alpha,
$$

and we set $\mathbb{D}^{\infty}=\bigcap_{p \geqslant 1} \bigcap_{k \in \mathbb{N}} \mathbb{D}^{k, p}$. Similarly, one can define, for $H=L^{2}\left(\Lambda_{T}\right)$, the spaces $\mathbb{D}^{k, p}(H)$ and $\mathbb{D}^{\infty}(H)$, and the related $\|\cdot\|_{k, p, H}$ norms (the related smooth functionals being of the form $F=\sum_{k=0}^{n} F_{k} v_{k}$ where $F_{k} \in \mathscr{S}$ and $v_{k} \in H$ ).

A random variable $F$ satisfies $(\mathrm{H} 3)$ if

$$
F \in \mathbb{D}^{\infty},\|D F\|^{-1} \in \bigcap_{p \geqslant 0} L^{p} .
$$

The derivation operator $D$ on $L^{2}(\Omega)$ has an adjoint called the Skorohod integral, denoted by $\delta$, and defined by the duality formula $\mathbb{E}[\delta u G]=\mathbb{E}[\langle u D G\rangle]$, where $\langle\cdot, \cdot\rangle$ denotes the usual scalar product in $L^{2}\left(\Lambda_{T}\right)$. We shall use the following properties of $D$ and $\delta$ : (here $U \in \mathbb{D}^{\infty}$, $X$ satisfies $(\mathrm{H} 3) ; f$ is a continuous function on $\mathbb{R}$ with polynomial growth and $F_{f}$ is a primitive of $f$ ).

$$
\delta \text { is continuous from } \mathbb{D}^{k+1, p}(H) \text { to } \mathbb{D}^{k, p} \text {. }
$$

$$
\mathbb{E}(U f(X))=\mathbb{E}\left(F_{f}(X) \delta\left(\frac{U D X}{\|D X\|^{2}}\right)\right) .
$$

(P1) is obtained via the Meyer inequalities, and (P2) is proved using the integration-byparts formula.

As stated in the introduction, we shall use in the sequel the following property of $\delta$ : if $u \in L^{2}\left(\Omega \times \Lambda_{T}\right)$ is an $\mathscr{F}_{t}$-adapted process, then $u$ is in the domain of $\delta$, and 
$\delta(u)=\int \Lambda_{T} \mathrm{U}_{x, t} W(\mathrm{~d} x, \mathrm{~d} t)$. The proof, in our context, is a mere adaptation of the original proof for the case of the Brownian motion on $\mathbb{R}$.

\subsection{Differentiability of $X(t, x)$}

Bally and Pardoux (1993) have proved that, under (H1) and (H2), $X(t, x)$ belongs to $\mathbb{D}^{\infty}$, and its Malliavin first-order derivative satisfies the following equation: for $r<t$,

$$
\begin{aligned}
D_{r, z} X(t, x)= & G_{t-r}(x, z) \varphi(X(r, z))+\int_{r}^{t} \int_{0}^{1} G_{t-s}(x, y) \varphi^{\prime}(X(s, y)) D_{r, z} X(s, y) W(\mathrm{~d} y, \mathrm{~d} s) \\
& +\int_{r}^{t} \int_{0}^{1} G_{t-s}(x, y) \psi^{\prime}(X(s, y)) D_{r, z} X(s, y) \mathrm{d} y \mathrm{~d} s
\end{aligned}
$$

(and $D_{r, z} X(t, x)=0$ when $\left.r>t\right)$.

The following result will be constantly used in the paper (cf. Bally and Pardoux (1993)).

\section{Proposition 3.1.}

(a) For all $M$, $p$, there exists a constant $C_{M, p}$ such that

$$
\sup _{(t, x) \in[0, T] \times[0,1]} \mathbb{E}\left\|D^{M} X(t, x)\right\|^{p} \leqslant C_{M, p}<\infty .
$$

(b) Let $\tau \in] 0, T[$. For all $p \in] 0,+\infty\left[\right.$ there exists a constant $C_{p, \tau}$ such that

$$
\mathbb{E}\left(\frac{1}{\|D X(t, x)\|^{p}}\right) \leqslant C_{p, \tau}<\infty .
$$

We remark that uniform pathwise estimates such as those stated by Nualart (1995, Theorem 2.2.1, p. 102) for stochastic differential equations cannot be obtained in our context, because of the presence of $G$ in (3.3).

\section{Proof of Theorem 2.1}

\subsection{The method}

As explained in the introduction, a standard method to obtain estimates for the density of $X(t, x)$ is to consider $\mathbb{E}[f(X(t, x)]$ for $f$ in a reasonably large functional vector space. More precisely, we prove in this section the following proposition.

Proposition 4.1. Let $\tau \in] 0, T\left[\right.$. There exists some real numbers $C_{\tau}$ and $C_{\tau, \epsilon}$ such that, for all $t \in[\tau, T], x \in[0,1], \epsilon \in] 0,1\left[\right.$ and $h$ such that $t+h \leqslant T$ and $x+h \leqslant 1$, for all $f \in C_{b}^{3}(\mathbb{R})$, and $F_{f}$, antiderivative of $f$, we have

$$
\mid \mathbb{E}\left(f(X(t+h, x))-\mathbb{E}\left(f(X(t, x)) \mid \leqslant C_{\tau}\left\|F_{f}\right\|_{\infty} h^{1 / 2},\right.\right.
$$




$$
\begin{gathered}
\mid \mathbb{E}\left(f(X(t, x+h))-\mathbb{E}\left(f(X(t, x)) \mid \leqslant C_{\tau, \epsilon}\left\|F_{f}\right\|_{\infty} h^{1-\epsilon},\right.\right. \\
\|\mathbb{E}[f(X(t, \cdot))]\|_{\infty} \leqslant C_{\tau}\left\|F_{f}\right\|_{\infty}, \\
\left|\mathbb{E}\left[\Delta_{h}^{2} f(X(t, \cdot))(x)\right]\right| \leqslant C_{\tau}\left\|F_{f}\right\|_{\infty} h .
\end{gathered}
$$

Supposing that Proposition 4.1 is proved, then let $\left\{f_{n, y}\right\}_{n}$ be a sequence of Gaussian kernels of mean $y$ and whose variance decreases towards zero with $n$. If $F_{n, y}$ is the antiderivative of $f_{n, y}$ such that $F_{n, y}(-\infty)=0$, then it is clear that $\sup _{n, y}\left\|F_{n, y}\right\|_{\infty}<\infty$ and, on the other hand, $\forall(t, x), \lim _{n \rightarrow \infty} \mathbb{E}\left[f_{n, y}(X(t, x))\right]=p_{t, x}(y)$. Hence, taking limits in (4.1)-(4.4), we get the inequalities of Theorem 2.1.

Now, as the Itô formula cannot be used on the evolution equation (1.1), a natural tool to obtain estimates (4.1)-(4.4) is a Taylor expansion with integral remainder. Terms involving $f, f^{\prime}, f^{\prime \prime}$ will then appear, which will have to be replaced by others involving $F_{f}$. A natural way to do so is the integration-by-parts formula (P2). Of course, such a method is relevant if and only if there is no loss of regularity w.r.t. $h$ along the way. This is the object of the general result and its corollary which we now state and prove.

Proposition 4.2. Let $Z_{1}, Z_{2} \in \mathbb{D}^{\infty}$ and $\xi_{1}, \xi_{2}$ be random variables satisfying (H3). Setting $\sigma_{i}=\left\|D \xi_{i}\right\|^{2}$, we define by induction (for $i=1,2$ )

$$
H_{0}\left(Z_{i} ; \xi_{i}\right)=Z_{i}, \quad H_{n+1}\left(Z_{i} ; \xi_{i}\right)=\delta\left(H_{n}\left(Z_{i} ; \xi_{i}\right) \frac{D \xi_{i}}{\sigma_{i}}\right) .
$$

Then, for all $n \geqslant 1, q \geqslant 1$ and all $p>1$,

$$
\left\|H_{n}\left(Z_{1} ; \xi_{1}\right)-H_{n}\left(Z_{2} ; \xi_{2}\right)\right\|_{q, p} \leqslant C_{p, q, n}\left(\left\|Z_{1}-Z_{2}\right\|_{q+n, 4^{n} p}+\left\|\xi_{1}-\xi_{2}\right\|_{q+2 n, 4^{n} p}\right),
$$

where $C_{p, q, n}=K_{p, q, n}\left\{\left\|Z_{2}\right\|_{q+2 n, 4^{n} p}+\sup _{i \in\{1,2\}}\left\{\left\|\xi_{i}\right\|_{q+2 n, 4^{n} p}+\mathbb{E}\left|\sigma_{i}^{-1}\right|^{\kappa_{n}}\right\}\right\}$, with a constant $K_{p, q, n}$ depending only on $p, q, n$ and an integer $\kappa_{n}$ depending only on $n$.

Proof. We show the result for $n=1$, the general case being obtained by induction. Because of (P1), there exists a constant $C_{q, p}$ depending only on $p, q$ such that

$$
\begin{aligned}
& \left\|H_{1}\left(Z_{1} ; \xi_{1}\right)-H_{1}\left(Z_{2} ; \xi_{2}\right)\right\|_{q, p} \\
& \leqslant\left\|\delta\left(\left(Z_{1}-Z_{2}\right) \frac{D \xi_{1}}{\sigma_{1}}\right)\right\|_{q, p}+\left\|\delta\left(\frac{Z_{2}}{\sigma_{1}} D\left(\xi_{1}-\xi_{2}\right)\right)\right\|_{q, p}+\left\|\delta\left(\frac{\sigma_{2}-\sigma_{1}}{\sigma_{1} \cdot \sigma_{2}} Z_{2} D \xi_{2}\right)\right\|_{q, p} \\
& \leqslant C_{q, p}\left(\left\|\left(Z_{1}-Z_{2}\right) \frac{D \xi_{1}}{\sigma_{1}}\right\|_{q+1, p, H}+\left\|\frac{Z_{2}}{\sigma_{1}} D\left(\xi_{1}-\xi_{2}\right)\right\|_{q+1, p, H}+\left\|\frac{\sigma_{2}-\sigma_{1}}{\sigma_{1} \cdot \sigma_{2}} Z_{2} D \xi_{2}\right\|_{q+1, p, H}\right) \\
& :=A_{1}+A_{2}+A_{3} .
\end{aligned}
$$

Using the Leibniz formula, the Schwarz inequality and the fact that the $\xi_{i}$ satisfy (H3), we obtain 


$$
\begin{aligned}
& A_{1} \leqslant C_{q, p}\left\|Z_{1}-Z_{2}\right\|_{q+1,2 p}\left\|\frac{D \xi_{1}}{\sigma_{1}}\right\|_{q+1,2 p, H} \leqslant C_{q, p}\left\|Z_{1}-Z_{2}\right\|_{q+1,2 p}, \\
& A_{2} \leqslant C_{q, p}\left\|\frac{Z_{2}}{\sigma_{1}}\right\|_{q+1,2 p}\left\|D\left(\xi_{1}-\xi_{2}\right)\right\|_{q+1,2 p, H} \leqslant C_{q, p}\left\|\xi_{1}-\xi_{2}\right\|_{q+2,2 p} .
\end{aligned}
$$

As for $A_{3}$, insofar as

$$
D_{\alpha}^{k}\left(\sigma_{2}-\sigma_{1}\right)=\int_{\Lambda_{T}} D_{\alpha}^{k}\left[\left\{D_{r, z}\left(\xi_{2}-\xi_{1}\right)\right\}\left\{D_{r, z}\left(\xi_{2}+\xi_{1}\right)\right\}\right] \mathrm{d} z \mathrm{~d} r
$$

we easily have, using again the Leibniz formula and the Schwarz inequality,

$$
\left\|\sigma_{2}-\sigma_{1}\right\|_{q+1,2 p} \leqslant K\left\|\xi_{2}-\xi_{1}\right\|_{q+2,4 p}
$$

which, proceeding as previously, yields

$$
A_{3} \leqslant C_{q, p}\left\|\xi_{2}-\xi_{1}\right\|_{q+2,4 p} .
$$

This concludes the proof.

From Proposition 4.2, we deduce the following.

Corollary 4.1. Let $f$ be in $C_{b}^{r_{0}}(\mathbb{R})$ and $F_{f}$ be an antiderivative of $f$. For $r \leqslant 3$, if $Z$ and $\xi$ are random variables such that $Z \in \mathbb{D}^{\infty}$ and $\xi$ satisfies (H3), then, for $r \leqslant r_{0}$,

$$
\left|\mathbb{E}\left[f^{(r)}(\xi) Z\right]\right| \leqslant C_{r}\left\|F_{f}\right\|_{\infty}\|Z\|_{r+1,2^{2 r+2}},
$$

where $C_{r}=K_{r}\left\{\|\xi\|_{2(r+1), 2^{2 r+2}}+\mathbb{E}\left|\sigma^{-1}\right|^{\kappa_{r}}\right\}$, with a constant $K_{r}$ depending only on $r$ and an integer $\kappa_{r}$ depending only on $r$.

Proof. Using (P2) $r+1$ times, we have

$$
\left|\mathbb{E}\left[f^{(r)}(\xi) Z\right]\right|=\left|\mathbb{E}\left[F_{f}(\xi) H_{r+1}(Z, \xi)\right]\right| \leqslant\left\|F_{f}\right\|_{\infty}\left\|H_{r+1}(Z ; \xi)\right\|_{0,1},
$$

where the random variables $H_{k}\left(Z_{i}, \xi\right)$ are those obtained via the construction of Proposition 4.2. Then it suffices to use Proposition 4.2 with $Z_{1}=Z, Z_{2}=0, \xi_{1}=\xi_{2}=\xi, q=0, p=1$ and $n=r+1$.

\subsection{Proof of estimate (4.1)}

Assume that $t \in[\tau, T], x \in[0,1]$ and $h>0$ with $t+h \leqslant T$. One has the following Taylor expansion: 


$$
\begin{aligned}
\mathbb{E}[f(X(t+h, x))-f(X(t, x))]= & \mathbb{E}\left[f^{\prime}(X(t, x))(X(t+h, x)-X(t, x))\right] \\
& +\mathbb{E}\left((X(t+h, x)-X(t, x))^{2} \int_{0}^{1}(1-v) f^{\prime \prime}\left(Y_{t, x}(h, v)\right) \mathrm{d} v\right) \\
& :=T_{1}+T_{2},
\end{aligned}
$$

where $Y_{t, x}(h, v)=X(t, x)+v(X(t+h, x)-X(t, x))$.

\subsubsection{Bound for $T_{1}$}

We have

$$
\begin{aligned}
T_{1}= & \mathbb{E}\left[f^{\prime}(X(t, x))\left\{G_{t+h}\left(x, X_{0}\right)-G_{t}\left(x, X_{0}\right)\right\}\right] \\
& +\mathbb{E}\left[f ^ { \prime } ( X ( t , x ) ) \left(\int_{0}^{t} \int_{0}^{1}\left\{G_{t+h-s}(x, y)-G_{t-s}(x, y)\right\} \psi(X(s, y)) \mathrm{d} y \mathrm{~d} s\right.\right. \\
& \left.\left.+\int_{t}^{t+h} \int_{0}^{1} G_{t+h-s}(x, y) \psi(X(s, y)) \mathrm{d} y \mathrm{~d} s\right)\right] \\
& +\mathbb{E}\left(f^{\prime}(X(t, x)) \int_{0}^{t} \int_{0}^{1}\left\{G_{t+h-s}(x, y)-G_{t-s}(x, y)\right\} \varphi(X(s, y)) W(\mathrm{~d} y, \mathrm{~d} s)\right) \\
& +\mathbb{E}\left(f^{\prime}(X(t, x)) \int_{t}^{t+h} \int_{0}^{1} G_{t+h-s}(x, y) \varphi(X(s, y)) W(\mathrm{~d} y, \mathrm{~d} s)\right) \\
:= & T_{11}+T_{12}+T_{13}+T_{14} .
\end{aligned}
$$

It is clear that $T_{14}=0$ since $f^{\prime}(X(t, x))$ is $\mathscr{F}_{t}$ measurable and

$$
M_{u}=\int_{0}^{u} \int_{0}^{1} G_{t+h-s}(x, y) \varphi(X(s, y)) W(\mathrm{~d} y, \mathrm{~d} s), \quad u \leqslant t+h,
$$

is an $\mathscr{F}_{u^{-}} L^{2}$-bounded martingale.

On the other hand, set $H_{t, x}^{(0)}(h)=G_{t+h}\left(x, X_{0}\right)-G_{t}\left(x, X_{0}\right)$. The function $t \rightarrow G_{t}\left(x, X_{0}\right)$ is clearly infinitely differentiable on $[\tau, T]$ and $\sup _{(t, x) \in[\tau, T] \times[0,1]}\left|(\partial / \partial t)\left\{G_{t}\left(x, X_{0}\right)\right\}\right| \leqslant C_{\tau}$. Therefore we have $\left|H_{t, x}^{(0)}(h)\right|^{p} \leqslant C_{p} h^{p}$ and, applying Corollary 4.1 with $Z=$ $G_{t+h}\left(x, X_{0}\right)-G_{t}\left(x, X_{0}\right)$ and $\xi=X(t, x)$, we get $\left|T_{11}\right| \leqslant C_{\tau}\left\|F_{f}\right\|_{\infty} h$. (Indeed, $Z$ being deterministic, its Malliavin derivatives are zero and, by Proposition 3.1, $X(t, x)$ satisfies (H3).)

We now turn to $T_{12}$. We must prove that 


$$
\begin{aligned}
U_{t, x}(h)= & \int_{0}^{t} \int_{0}^{1}\left\{G_{t+h-s}(x, y)-G_{t-s}(x, y)\right\} \psi(X(s, y)) \mathrm{d} y \mathrm{~d} s \\
& +\int_{t}^{t+h} \int_{0}^{1} G_{t+h-s}(x, y) \psi(X(s, y)) \mathrm{d} y \mathrm{~d} s
\end{aligned}
$$

is in $\mathbb{D}^{\infty}$ with, for all $p, q, \sup _{t, x}\left\|U_{t, x}(h)\right\|_{q, p} \leqslant C_{p} h^{1 / 2}$ in order to apply Corollary 4.1 with $Z=U_{t, x}(h)$ and $\xi=X(t, x)$. For $p>1$, using that $\psi$ is bounded, we have

$$
\begin{aligned}
\mathbb{E}\left[\left|U_{t, x}(h)\right|^{p}\right] \leqslant & C_{p}\left[\left(\int_{0}^{t} \int_{0}^{1}\left|G_{t+h-s}(x, y)-G_{t-s}(x, y)\right| \mathrm{d} y \mathrm{~d} s\right)^{p}\right. \\
& \left.+\left(\int_{t}^{t+h} \int_{0}^{1} G_{t+h-s}(x, y) \mathrm{d} y \mathrm{~d} s\right)^{p}\right] .
\end{aligned}
$$

We then use Lemma A.1.1; taking $\beta=\frac{3}{2}$ in (c), we get $\mathbb{E}\left|U_{t, x}(h)\right|^{p} \leqslant C_{p} h^{p / 2}$. We now have to bound the derivatives of $U_{t, x}(h)$. We do so for the first order; the general case is similarly dealt with using the Leibniz formula, the fact that $\varphi$ and $\psi$ are uniformly bounded and Proposition 3.1. We have, for $r<t+h$ and $z \in[0,1]$,

$$
\begin{aligned}
D_{r, z} U_{t, x}(h)= & \int_{0}^{t} \int_{0}^{1}\left\{G_{t+h-s}(x, y)-G_{t-s}(x, y)\right\} \psi^{\prime}(X(s, y)) D_{r, z} X(s, y) \mathrm{d} y \mathrm{~d} s \\
& +\int_{t}^{t+h} \int_{0}^{1} G_{t+h-s}(x, y) \psi^{\prime}(X(s, y)) D_{r, z} X(s, y) \mathrm{d} y \mathrm{~d} s,
\end{aligned}
$$

(this quantity being zero when $r>t+h$ ). Using the Hölder inequality w.r.t. $\left|G_{t+h-s}(x, y)-\right| G_{t-s}(x, y) \mid \mathrm{d} y \mathrm{~d} s$ (or $G_{t+h-s}(x, y) \mathrm{d} y \mathrm{~d} s$ ), we easily obtain

$$
\begin{aligned}
\left\|D U_{t, x}(h)\right\|^{p} \leqslant & C_{p}\left\{\left(\int_{0}^{t} \int_{0}^{1}\left|G_{t+h-s}(x, y)-G_{t-s}(x, y)\right| \mathrm{d} y \mathrm{~d} s\right)^{p-1}\right. \\
& \times G \int_{0}^{t} \int_{0}^{1}\left|G_{t+h-s}(x, y)-G_{t-s}(x, y)\right|\|D X(s, y)\|^{p} \mathrm{~d} y \mathrm{~d} s \\
& \left.+\left(\int_{t}^{t+h} \int_{0}^{1} G_{t+h-s}(x, y) \mathrm{d} y \mathrm{~d} s\right)^{p-1} \int_{t}^{t+h} \int_{0}^{1} G_{t+h-s}(x, y)\|D X(s, y)\|^{p} \mathrm{~d} y \mathrm{~d} s\right\},
\end{aligned}
$$

which clearly gives, taking expectations and using Proposition 3.1, $\mathbb{E}\left\|D U_{t, x}(h)\right\|^{p} \leqslant C_{p} h^{p / 2}$.

We now turn to $T_{13}$, which is the most difficult term because of the stochastic integral, the main source of loss of regularity. Insofar as the integrand is adapted, this term can be viewed as a Skorohod integral and the duality formula between $\delta$ and $D$ yields 


$$
T_{13}=\mathbb{E}\left(f^{\prime \prime}(X(t, x)) \int_{0}^{t} \int_{0}^{1} D_{s, y} X(t, x)\left\{G_{t+h-s}(x, y)-G_{t-s}(x, y)\right\} \varphi(X(s, y)) \mathrm{d} y \mathrm{~d} s\right) .
$$

For $(\bar{t}, \bar{x}) \in[\tau, T] \times[0,1]$ such that $\bar{t} \geqslant t$, set

$$
B_{r, z}(\bar{t}, \bar{x})=\left(G_{\dot{t}+h-r}(\bar{x}, y)-G_{\dot{t}-r}(\bar{x}, y)\right) \varphi(X(r, z)), \quad(r, z) \in \Lambda_{t} .
$$

Because of (3.3), one has (remember that $\langle\cdot, \cdot\rangle$ denotes the usual scalar product on $L^{2}\left(\Lambda_{T}\right)$ ):

$$
\begin{aligned}
\langle D X(t, x), B(\bar{t}, \bar{x})\rangle= & \left\langle G_{t-} .(x, \cdot) \varphi(X(\cdot, \cdot)), B(\bar{t}, \bar{x})\right\rangle \\
& +\int_{0}^{t} \int_{0}^{1} G_{t-s}(x, y) \varphi^{\prime}(X(s, y))\langle D X(s, y), B(\bar{t}, \bar{x})\rangle W(\mathrm{~d} y, \mathrm{~d} s) \\
& +\int_{0}^{t} \int_{0}^{1} G_{t-s}(x, y) \psi^{\prime}(X(s, y))\langle D X(s, y), B(\bar{t}, \bar{x})\rangle \mathrm{d} y \mathrm{~d} s .
\end{aligned}
$$

Then, using the Burkholder inequality and the Gronwall lemma, we easily get

$$
\mathbb{E}|\langle D X(t, x), B(\bar{t}, \bar{x})\rangle|^{p} \leqslant C_{p, \tau} \mathbb{E} \mid\left\langle\left. G_{t-\cdot}(x, \cdot) \varphi(X(\cdot, \cdot), B(\bar{t}, \bar{x})\rangle\right|^{p},\right.
$$

where the constant $C_{p, \tau}$ does not depend on $(\bar{t}, \bar{x})$. Therefore, setting $T_{13}=$ $\mathbb{E}\left[f^{\prime \prime}(X(t, x)) S_{t, x}(h)\right]$, we have

$$
\mathbb{E}\left|S_{t, x}(h)\right|^{p} \leqslant C_{p, \tau} \mathbb{E}\left|\int_{0}^{t} \int_{0}^{1} G_{t-s}(x, y)\left\{G_{t+h-s}(x, y)-G_{t-s}(x, y)\right\} \varphi^{2}(X(s, y)) \mathrm{d} y \mathrm{~d} s\right|^{p} .
$$

Therefore the regularity w.r.t. $h$ of $S_{t, x}(h)$ is determined by that of

$$
\int_{0}^{t} \int_{0}^{1} G_{t-s}(x, y)\left|G_{t+h-s}(x, y)-G_{t-s}(x, y)\right| \mathrm{d} y \mathrm{~d} s,
$$

and, using Lemma A1.3, we finally get

$$
\mathbb{E}\left|S_{t, x}(h)\right|^{p} \leqslant C_{p, \tau} h^{p / 2} .
$$

As for the derivatives of $S_{t, x}(h)$, they are similarly handled by differentiating (4.5) and using the methods exposed above. Therefore we can apply Corollary 4.1 and thus obtain $\left|T_{13}\right| \leqslant C_{\tau}\left\|F_{f}\right\|_{\infty} h^{1 / 2}$.

\subsubsection{Bound for $T_{2}$}

We have $T_{2}=\mathbb{E}\left[\{X(t+h, x)-X(t, x)\}^{2} \int_{0}^{1}(1-v) f^{\prime \prime}\left(Y_{t, x}(h, v)\right) \mathrm{d} v\right]$. In order to apply Corollary 4.1 with $\xi=Y_{t, x}(h, v), Z=\{X(t+h, x)-X(t, x)\}^{2}$, we only have to prove the following lemma.

Lemma 4.1. $Y_{t, x}(h, v)$ satisfy (H3) uniformly in $(h, v)$, i.e. 


$$
\begin{aligned}
\forall \tau \in] 0, T\left[, \forall p>1, \sup _{(t, x, h, v) / \tau \leqslant t \leqslant t+h \leqslant T,(x, v) \in[0,1]^{2}}\left[\mathbb{E}\left\{\left\|D Y_{t, x}(h, v)\right\|^{p}+\left\|D Y_{t, x}(h, v)\right\|^{-p}\right\}\right]\right. & \\
& \leqslant C_{p, \tau}<\infty .
\end{aligned}
$$

Indeed, if this result holds, the required bound for $T_{2}$ is obtained by applying three times the integration-by-parts formula (P2), since $\left\|(X(t+h, x)-X(t, x))^{2}\right\|_{q, p}$ clearly is of order $h^{1 / 2}$.

So as to prove Lemma 4.1, we first show the following auxiliary result, which studies the regularity of the function $t \mapsto \mathbb{E}\left|\int_{0}^{t} \int_{0}^{1}\left(D_{r, z} X(s, y)\right)^{2} \mathrm{~d} z \mathrm{~d} r\right|^{p}$, i.e. to some extent the behaviour of $D X(t, x)$ in the neighbourhood of $t$, not pathwise but from a Hilbertian point of view.

Lemma 4.2. Let $\eta$ be a real number such that $t-\eta>0$. For all $p>1$ there exists a constant $C_{p}$ such that, for all $t>0, s \geqslant t, y \in[0,1]$,

$$
\mathbb{E}\left|\int_{t-\eta}^{t} \int_{0}^{1}\left(D_{r, z} X(s, y)\right)^{2} \mathrm{~d} z \mathrm{~d} r\right|^{p} \leqslant C_{p} \eta^{p / 2} \text {. }
$$

Proof of Lemma 4.2. We define

$$
H(s, y):=\mathbb{E}\left|\int_{t-\eta}^{t} \int_{0}^{1}\left\{D_{r, z} X(s, y)\right\}^{2} \mathrm{~d} z \mathrm{~d} r\right|^{p}, \quad H(s)=\sup _{y} H(s, y), \quad K_{t}(s)=\sup _{t \leqslant v \leqslant s} H(v) .
$$

Because of Proposition 3.1, we know that $K_{t}(s)$ is uniformly bounded with respect to $\eta$, $s$ and $t \in[0, T]$. On the other hand, since $\varphi$ is uniformly bounded, we have

$$
\begin{aligned}
H(s, y) \leqslant & C_{p}\left\{\left(\int_{t-\eta}^{t} \int_{0}^{1} G_{s-r}^{2}(y, z) \mathrm{d} z \mathrm{~d} r\right)^{p}\right. \\
& +\mathbb{E}\left|\int_{t-\eta}^{t} \int_{0}^{1}\left(\int_{r}^{s} \int_{0}^{1} G_{s-v}(y, u) \varphi^{\prime}(X(v, u)) D_{r, z} X(v, u) W(\mathrm{~d} v, \mathrm{~d} u)\right)^{2} \mathrm{~d} z \mathrm{~d} r\right|^{p} \\
& \left.+\mathbb{E}\left|\int_{t-\eta}^{t} \int_{0}^{1}\left(\int_{r}^{s} \int_{0}^{1} G_{s-v}(y, u) \psi^{\prime}(X(v, u)) D_{r, z} X(v, u) \mathrm{d} v \mathrm{~d} u\right)^{2} \mathrm{~d} z \mathrm{~d} r\right|^{p}\right\} \\
:= & A_{1}+A_{2}+A_{3} .
\end{aligned}
$$

It is easy to see that $A_{1} \leqslant C_{p} \eta^{p / 2}$. As for $A_{2}$, we use the following version of the Burkholder inequality for Hilbert-space valued martingales: if $\left(Q_{s, y}\right)_{(s, y) \in \Lambda_{T}}$ is a $L^{2}\left(\Lambda_{t}\right)$-valued predictable process, then

$$
\mathbb{E}\left|\int_{\Lambda_{t}}\left(\int_{0}^{t} \int_{0}^{1} Q_{v, u}(r, z) W(\mathrm{~d} v, \mathrm{~d} u)\right)^{2} \mathrm{~d} z \mathrm{~d} r\right|^{p} \leqslant C_{p} \mathbb{E}\left|\int_{0}^{t} \int_{0}^{1}\left(\int_{\Lambda_{t}} Q_{v, u}^{2}(r, z) \mathrm{d} z \mathrm{~d} r\right) \mathrm{d} v \mathrm{~d} u\right|^{p} .
$$

We then have 


$$
\begin{aligned}
A_{2} \leqslant & C_{p}\left\{\mathbb{E}\left|\int_{t-\eta}^{t} \int_{0}^{1}\left(\int_{t-\eta}^{v} \int_{0}^{1} G_{s-v}^{2}(y, u)\left(D_{r, z} X(v, u)\right)^{2} \mathrm{~d} z \mathrm{~d} r\right) \mathrm{d} v \mathrm{~d} u\right|^{p}\right. \\
& \left.+\mathbb{E}\left|\int_{t}^{s} \int_{0}^{1}\left(\int_{t-\eta}^{t} \int_{0}^{1} G_{s-v}^{2}(y, u)\left\{D_{r, z} X(v, u)\right\}^{2} \mathrm{~d} z \mathrm{~d} r\right) \mathrm{d} v \mathrm{~d} u\right|^{p}\right\} \\
:= & C_{p}\left(A_{21}+A_{22}\right)
\end{aligned}
$$

and, using the Hölder inequality w.r.t. the suitable measure, we obtain

$$
\begin{aligned}
A_{21} \leqslant & \int_{t-\eta}^{t} \int_{0}^{1} G_{s-v}^{2}(y, u) \sup _{u, v}\left(\mathbb{E}\left|\int_{0}^{T} \int_{0}^{1}\left(D_{r, z} X(v, u)\right)^{2} \mathrm{~d} z \mathrm{~d} r\right|^{p}\right) \mathrm{d} v \mathrm{~d} u \\
& \times\left(\int_{t-\eta}^{t} \int_{0}^{1} G_{s-v}^{2}(y, u) \mathrm{d} u \mathrm{~d} v\right)^{p-1} \\
\leqslant & C_{p} \eta^{p / 2}
\end{aligned}
$$

As for $A_{22}$, using the same method, we get $A_{22} \leqslant C_{p} \int_{t}^{s} K_{t}(v) \mathrm{d} v$. Finally we bound $A_{3}$ because of the Hölder inequality and the methods employed in the treatment of $A_{2}$. Then the Gronwall lemma yields the result.

Proof of Lemma 4.1. By means of Lemma 2.33.1 of Nualart (1995), we only have to prove the following estimate: for all $\tau \in] 0, T$, there exist $\lambda>0$ and $\epsilon_{0}(\tau)$ such that for all $q$ and all $0<\epsilon \leqslant \epsilon_{0}(\tau)$ we have

(ND)

$$
\sup _{v} \mathbb{P}\left(\left\|D Y_{t, x}(h, v)\right\|^{2} \leqslant \epsilon\right) \leqslant C_{q, \tau} \epsilon^{\lambda q} .
$$

To prove (ND), we shall write

$$
Y_{t, x}(h, v)=Z_{t, x}(h, v)+v\left\{X(t+h, x)-G_{h}(x, X(t, \cdot))\right\},
$$

where $Z_{t, x}(h, v)=X(t, x)+v\left\{G_{h}(x, X(t, \cdot))-X(t, x)\right\}$. Then, for $\left.\eta \in\right] 0, \tau[$,

$$
\begin{aligned}
\left\|D Y_{t, x}(h, v)\right\|^{2} \geqslant & \int_{t-\eta}^{t} \int_{0}^{1}\left(D_{r, z} Y_{t, x}(h, v)\right)^{2} \mathrm{~d} r \mathrm{~d} z \\
\geqslant & \frac{1}{2} \int_{t-\eta}^{t} \int_{0}^{1}\left(D_{r, z} Z_{t, x}(h, v)\right)^{2} \mathrm{~d} r \mathrm{~d} z-v^{2} \\
& \times \int_{t-\eta}^{t} \int_{0}^{1}\left[D_{r, z}\left\{X(t+h, x)-G_{h}(x, X(t, \cdot))\right\}\right]^{2} \mathrm{~d} r \mathrm{~d} z
\end{aligned}
$$

and we have 


$$
\begin{aligned}
\mathbb{P}\left(\left\|D Y_{t, x}(h, v)\right\|^{2} \leqslant \epsilon\right) \leqslant & \mathbb{P}\left(\int_{t-\eta}^{t} \int_{0}^{1}\left\{D_{r, z} Z_{t, x}(h, v)\right\}^{2} \mathrm{~d} r \mathrm{~d} z \leqslant 4 \epsilon\right) \\
& +\mathbb{P}\left(v^{2} \int_{t-\eta}^{t} \int_{0}^{1}\left[D_{r, z}\left\{X(t+h, x)-G_{h}(x, X(t, \cdot))\right\}\right]^{2} \mathrm{~d} r \mathrm{~d} z \geqslant \epsilon\right) .
\end{aligned}
$$

We first deal with $Z_{t, x}(h, v)$ :

$$
\begin{aligned}
D_{r, z} Z_{t, x}(h, v)= & G_{t-r}(x, z) \varphi(X(r, z))+\int_{r}^{t} \int_{0}^{1} G_{t-s}(x, y) \psi^{\prime}(X(s, y)) D_{r, z} X(s, y) \mathrm{d} y \mathrm{~d} s \\
& +\int_{r}^{t} \int_{0}^{1} G_{t-s}(x, y) \varphi^{\prime}(X(s, y)) D_{r, z} X(s, y) W(\mathrm{~d} y, \mathrm{~d} s) \\
& +v\left(\left\{G_{t+h-r}(x, z)-G_{t-r}(x, z)\right\} \varphi(X(r, z))\right. \\
& +\int_{r}^{t} \int_{0}^{1}\left\{G_{t+h-s}(x, y)-G_{t-s}(x, y)\right\} \psi^{\prime}(X(s, y)) D_{r, z} X(s, y) \mathrm{d} y \mathrm{~d} s \\
& \left.+\int_{r}^{t} \int_{0}^{1}\left\{G_{t+h-s}(x, y)-G_{t-s}(x, y)\right\} \varphi^{\prime}(X(s, y)) D_{r, z} X(s, y) W(\mathrm{~d} y, \mathrm{~d} s)\right) \\
= & G_{t-r}(x, z) \varphi(X(r, z))+\sum_{k=1}^{5} I_{k} .
\end{aligned}
$$

Then using the strong ellipticity hypothesis (H2) we get

$$
\int_{t-\eta}^{t} \int_{0}^{1}\left(D_{r, z} Z_{t, x}(h, v)\right)^{2} \mathrm{~d} z \mathrm{~d} r \geqslant \frac{c^{2}}{2} \int_{t-\eta}^{t} \int_{0}^{1} G_{t-r}^{2}(x, z) \mathrm{d} z \mathrm{~d} r-\int_{t-\eta}^{t} \int_{0}^{1}\left(\sum_{k=1}^{5} I_{k}\right)^{2} \mathrm{~d} z \mathrm{~d} r,
$$

and the first term is bounded from below by $C \eta^{1 / 2}$ owing to Lemma A1.2 of Appendix 1 . Hence

$$
\mathbb{P}\left(\int_{t-\eta}^{t} \int_{0}^{1}\left\{D_{r, z} Z_{t, x}(h, v)\right\}^{2} \mathrm{~d} r \mathrm{~d} z \leqslant \epsilon\right) \leqslant \mathbb{P}\left\{\int_{t-\eta}^{t} \int_{0}^{1}\left(\sum_{k=1}^{5} I_{k}\right)^{2} \mathrm{~d} z \mathrm{~d} r \geqslant C \eta^{1 / 2}-\epsilon\right\} .
$$

We choose $\eta=\epsilon^{3(1-\delta)}$. If $\delta>\frac{1}{3}$, then there exists $\epsilon_{0}(\tau)$ such that, for all $\epsilon \leqslant \epsilon_{0}(\tau)$, we have $C \eta^{1 / 2}-\epsilon \geqslant \epsilon / 2$. Using the Chebyshev inequality for $q>1$,

$$
\begin{aligned}
\mathbb{P}\left(\int_{t-\eta}^{t} \int_{0}^{1}\left(D_{r, z} Z_{t, x}(h, v)\right)^{2} \mathrm{~d} z \mathrm{~d} r \leqslant \epsilon\right) & \leqslant \frac{C_{q}}{\epsilon^{q}} \mathbb{E}\left|\int_{t-\eta}^{t} \int_{0}^{1}\left(\sum_{k=1}^{5} I_{k}\right)^{2} \mathrm{~d} z \mathrm{~d} r\right|^{q} \\
& \leqslant \frac{C_{q}}{\epsilon^{q}} \sum_{k=1}^{5} \mathbb{E}\left|\int_{t-\eta}^{t} \int_{0}^{1} I_{k}^{2} \mathrm{~d} z \mathrm{~d} r\right|^{q}
\end{aligned}
$$


Then, using the Burkholder-Davis-Gundy inequalities for Hilbert-valued martingales (4.6) and Lemma 4.2, taking into account that $v \leqslant 1$, it is easy to see that

$$
\forall k \leqslant 5, \mathbb{E}\left|\int_{t-\eta}^{t} \int_{0}^{1} I_{k}^{2} \mathrm{~d} z \mathrm{~d} r\right|^{q} \leqslant C_{q, \tau} \eta^{q} .
$$

Indeed, for instance, using (4.6) and the boundedness of $\varphi$ :

$$
\begin{aligned}
& \mathbb{E}\left|\int_{t-\eta}^{t} \int_{0}^{1} I_{5}^{2} \mathrm{~d} z \mathrm{~d} r\right|^{q} \\
&=\mathbb{E}\left|\int_{t-\eta}^{t} \int_{0}^{1}\left(\int_{r}^{t} \int_{0}^{1}\left\{G_{t+h-s}(x, y)-G_{t-s}(x, y)\right\} \varphi^{\prime}(X(s, y)) D_{r, z} X(s, y) W(\mathrm{~d} y, \mathrm{~d} s)\right)^{2} \mathrm{~d} r \mathrm{~d} z\right|^{q} \\
& \leqslant C\left(\int_{t-\eta}^{t} \int_{0}^{1}\left\{G_{t+h-s}(x, y)-G_{t-s}(x, y)\right\}^{2} \mathrm{~d} y \mathrm{~d} s\right)^{q-1} \\
& \times \int_{t-\eta}^{t} \int_{0}^{1}\left\{G_{t+h-s}(x, y)-G_{t-s}(x, y)\right\}^{2} \mathbb{E}\left|\int_{t-\eta}^{s} \int_{0}^{1}\left\{D_{r, z} X(s, y)\right\}^{2} \mathrm{~d} z \mathrm{~d} r\right|^{q} \mathrm{~d} y \mathrm{~d} s \\
& \leqslant C_{q \tau}\left(\eta^{1 / 2}\right)^{q-1} \eta^{q / 2} \int_{t-\eta}^{t} \int_{0}^{1}\left\{G_{t+h-s}(x, y)-G_{t-s}(x, y)\right\}^{2} \mathrm{~d} y \mathrm{~d} s \\
& \leqslant C_{q \tau} \eta^{q} .
\end{aligned}
$$

Hence, insofar as $\eta=\epsilon^{3(1-\delta)}$, we get a bound of order $(\eta / \epsilon)^{q}$, and $\eta / \epsilon=\epsilon^{3(1-\delta)-1}=\epsilon^{2-3 \delta}$. Therefore, provided that we choose $\delta \in] \frac{1}{3}, \frac{2}{3}[$, there exists $\beta>0$ such that, for all $q>1$,

$$
\mathbb{P}\left(\int_{t-\eta}^{t} \int_{0}^{1}\left\{D_{r, z} Z_{t, x}(h, v)\right\}^{2} \mathrm{~d} r \mathrm{~d} z \leqslant \epsilon\right) \leqslant C_{q \tau} \epsilon^{\beta q} .
$$

We now deal with the other term. On $[0, t]$, we have

$$
\begin{aligned}
D_{r, z}\left\{X(t+h, x)-G_{h}(x, X(t, \cdot))\right\}= & \int_{t}^{t+h} \int_{0}^{1} G_{t+h-s}(x, y) \varphi^{\prime}(X(s, y)) D_{r, z} X(s, y) W(\mathrm{~d} y, \mathrm{~d} s) \\
& +\int_{t}^{t+h} \int_{0}^{1} G_{t+h-s}(x, y) \psi^{\prime}(X(s, y)) D_{r, z} X(s, y) \mathrm{d} y \mathrm{~d} s .
\end{aligned}
$$

Using again the Chebyshev and the Burkholder-Davis-Gundy inequalities, as well as Lemma 4.2, we easily obtain

$\mathbb{P}\left(v^{2} \int_{t-\eta}^{t} \int_{0}^{1}\left[D_{r, z}\left\{X(t+h, x)-G_{h}(x, X(t, \cdot))\right\}\right]^{2} \mathrm{~d} r \mathrm{~d} z \geqslant \epsilon\right)$ 


$$
\leqslant C_{q \tau} \frac{v^{2 q}}{\epsilon^{q}} \eta^{q / 2}\left(\int_{t}^{t+h} \int_{0}^{1} G_{t+h-s}^{2}(x, y) \mathrm{d} y \mathrm{~d} s\right)^{q}
$$

Therefore, we see that several situations may occur.

(1) If $v \leqslant \epsilon^{\alpha}$, with $\alpha>0$ to be determined afterwards,

$$
\mathbb{P}\left(v^{2} \int_{t-\eta}^{t} \int_{0}^{1}\left[D_{r, z}\left\{X(t+h, x)-G_{h}(x, X(t, \cdot))\right\}\right]^{2} \mathrm{~d} r \mathrm{~d} z \geqslant \epsilon\right) \leqslant C_{q \tau}\left(\frac{\epsilon^{2 \alpha} \eta^{1 / 2}}{\epsilon}\right)^{q},
$$

and $\epsilon^{2 \alpha} \eta^{1 / 2} / \epsilon=\epsilon^{2 \alpha+1 / 2-3 \delta / 2}$, which imposes $\delta<(1+4 \alpha) / 3$; this restriction is consistent with $\delta \in] \frac{1}{3}, \frac{2}{3}\left[\right.$ provided that $(1+4 \alpha) / 3<\frac{2}{3}$, i.e. $\left.\alpha \in\right] 0, \frac{1}{4}[$.

(2) Hence we now fix such an $\alpha$ and study the case $v \geqslant \epsilon^{\alpha}$. We then have to distinguish two subcases, whether $h$ is "small" or not, because of the integral in (4.7). Then let $\eta^{\prime}>0$, $\eta^{\prime}=\epsilon^{3\left(1-\delta^{\prime}\right)}$, with $\delta^{\prime}$ a priori different from $\delta$.

(a) If $h<\eta^{\prime}$, we have, as before, with $\eta^{\prime}$ instead of $\eta$

$$
\mathbb{P}\left(\int_{t-\eta^{\prime}}^{t} \int_{0}^{1}\left\{D_{r, z} Z_{t, x}(h, v)\right\}^{2} \mathrm{~d} z \mathrm{~d} r \leqslant \epsilon\right) \leqslant C_{q}\left(\frac{\eta^{\prime}}{\epsilon}\right)^{q},
$$

which, as seen before, imposes that $\left.\delta^{\prime} \in\right] \frac{1}{3}, \frac{2}{3}[$. On the other hand, bounding $v$ by 1 in (4.7) and using Lemma A1.1 (c) of Appendix 1, we get

$$
\begin{aligned}
\mathbb{P}\left(v^{2} \int_{t-\eta^{\prime}}^{t} \int_{0}^{1}\left[D_{r, z}\left\{X(t+h, x)-G_{h}(x, X(t, \cdot))\right\}\right]^{2} \mathrm{~d} r \mathrm{~d} z \geqslant \epsilon\right) & \leqslant C_{q \tau}\left(\frac{\left(\eta^{\prime}\right)^{1 / 2}}{\epsilon}\right)^{q} h^{q / 2} \\
& \leqslant C_{q \tau}\left(\frac{\eta^{\prime}}{\epsilon}\right)^{q},
\end{aligned}
$$

since $h<\eta^{\prime}$, which therefore gives the same bound as before.

(b) Hence there remains to deal with the case $h \geqslant \eta^{\prime}$. Now, the expression involving $Z_{t, x}(h, v)$ is no longer relevant, and we proceed as follows: insofar as $t+h-\eta^{\prime} \geqslant t$, we "localize" our study near $t+h$. More precisely,

$$
\left\|D Y_{t, x}(h, v)\right\|^{2} \geqslant \int_{t+h-\eta^{\prime}}^{t+h} \int_{0}^{1}\left\{D_{r, z} Y_{t, x}(h, v)\right\}^{2} \mathrm{~d} z \mathrm{~d} r .
$$

As $Y_{t, x}(h, v)=X(t, x)+v\{X(t+h, x)-X(t, x)\}$, we have, for $r \in[t, t+h[$,

$$
D_{r, z} Y_{t, x}(h, v)=v D_{r, z} X(t+h, x),
$$

which implies, using on the one hand the evolution equation for $D_{r, x} X(t+h, x)$, and on the other hand the fact that $v \geqslant \epsilon^{\alpha}$ as well as (H2), that

$$
\left\|D Y_{t, x}(h, v)\right\|^{2} \geqslant \epsilon^{2 \alpha}\left\{\frac{c^{2}}{2} \int_{t+h-\eta^{\prime}}^{t+h} \int_{0}^{1} G_{t+h-r}^{2}(x, z) \mathrm{d} z \mathrm{~d} r-\int_{t+h-\eta^{\prime}}^{t+h} \int_{0}^{1}\left(\sum_{k=1}^{2} J_{k}\right)^{2} \mathrm{~d} z \mathrm{~d} r\right\},
$$

where 


$$
J_{1}=\int_{r}^{t+h} \int_{0}^{1} G_{t+h-s}(x, y) \varphi^{\prime}(X(s, y)) D_{r, z} X(s, y) W(\mathrm{~d} y, \mathrm{~d} s),
$$

and $J_{2}$ is the corresponding term with $\psi^{\prime}$. We then have

$$
\mathbb{P}\left(\left\|D Y_{t, x}(h, v)\right\|^{2} \leqslant \epsilon\right) \leqslant \mathbb{P}\left\{\epsilon^{2 \alpha} \int_{t+h-\eta^{\prime}}^{t+h} \int_{0}^{1}\left(\sum_{k=1}^{2} J_{k}\right)^{2} \mathrm{~d} z \mathrm{~d} r \geqslant C \epsilon^{2 \alpha}\left(\eta^{\prime}\right)^{1 / 2}-\epsilon\right\} .
$$

In order that there exists $\epsilon_{1}(\tau)<\epsilon_{0}(\tau)$ such that, for all $\epsilon \leqslant \epsilon_{1}(\tau)$ we have $C \epsilon^{2 \alpha}\left(\eta^{\prime}\right)^{1 / 2}-\epsilon \geqslant \epsilon / 2$, it is necessary and sufficient that $2 \alpha+\frac{1}{2}-\frac{3}{2} \delta^{\prime}<0$, which imposes that $\delta^{\prime}>(1+4 \alpha) / 3$. Choosing such a $\delta^{\prime}$, we then get, using the Chebyshev inequality,

$$
\mathbb{P}\left(\left\|D Y_{t, x}(h, v)\right\|^{2} \leqslant \epsilon\right) \leqslant \frac{C_{q}}{\epsilon^{q}} \mathbb{E}\left|\epsilon^{2 \alpha} \int_{t+h-\eta^{\prime}}^{t+h} \int_{0}^{1}\left(\sum_{k=1}^{2} J_{k}\right)^{2} \mathrm{~d} z \mathrm{~d} r\right|^{q},
$$

and, proceeding as for the evaluation of the moments of $I_{5}$,

$$
\mathbb{E}\left|\epsilon^{2 \alpha} \int_{t+h-\eta^{\prime}}^{t+h} \int_{0}^{1} J_{1}^{2} \mathrm{~d} z \mathrm{~d} r\right|^{q} \leqslant C_{q \tau} \epsilon^{2 \alpha q} \eta^{\prime q} \leqslant C_{q \tau} \eta^{\prime q} .
$$

Therefore we have again a bound of order $\left(\eta^{\prime} / \epsilon\right)^{q}$, which imposes the $\left.\delta^{\prime} \in\right](1+4 \alpha) / 3, \frac{2}{3}[$, restriction which is consistent with $\alpha \in] 0, \frac{1}{4}[$; this completes the proof.

\subsection{Proof of estimate (4.2)}

As in Section 4.2, we write $\mathbb{E}[f(X(t, x+h))-f(X(t, x))]=T_{1}+T_{2}$, with

$$
\begin{aligned}
& T_{1}=\mathbb{E}\left[f^{\prime}(X(t, x))\{X(t, x+h)-X(t, x)\}\right], \\
& T_{2}=\mathbb{E}\left(\{X(t, x+h)-X(t, x)\}^{2} \int_{0}^{1}(1-v) f^{\prime \prime}\left(R_{t, x, h}(v)\right) \mathrm{d} v\right),
\end{aligned}
$$

where $R_{t, x, h}(v)=X(t, x)+v\{X(t, x+h)-X(t, x)\}$. We proceed exactly as for the time regularity. As before, $T_{2}$ is easily dealt with, owing to Proposition 4.2, via the following lemma. (Its proof, very similar to that of Lemma 4.1, is omitted.)

Lemma 4.3. $R_{t, x}(h, v)$ satisfy $(\mathrm{H} 3)$ uniformly in $(h, v)$, i.e.

$$
\begin{aligned}
\forall \tau \in] 0, T\left[, \forall p>1, \sup _{(t, x, h, v) / \tau \leqslant t \leqslant t+h \leqslant T,(x, v) \in[0,1]^{2}}\left[\mathbb{E}\left\{\left\|D R_{t, x}(h, v)\right\|^{p}+\left\|D R_{t, x}(h, v)\right\|^{-p}\right\}\right]\right. & \\
& \leqslant C_{\tau, p}<\infty .
\end{aligned}
$$

Hence we now have to prove that $\left|T_{1}\right| \leqslant C_{\epsilon}\left\|F_{f}\right\|_{\infty} h^{1-\epsilon}$. We then write $T_{1}=T_{11}+$ $T_{12}+T_{13}$, with 


$$
\begin{aligned}
& T_{11}=\mathbb{E}\left[f^{\prime}(X(t, x))\left\{G_{t}\left(x+h, X_{0}\right)-G_{t}\left(x, X_{0}\right)\right\}\right], \\
& T_{12}=\mathbb{E}\left\{f^{\prime}(X(t, x))\left(\int_{0}^{t} \int_{0}^{1}\left\{G_{t-s}(x+h, y)-G_{t-s}(x, y)\right\} \varphi(X(s, y)) W(\mathrm{~d} y, \mathrm{~d} s)\right)\right\}, \\
& T_{13}=\mathbb{E}\left\{f^{\prime}(X(t, x))\left(\int_{0}^{t} \int_{0}^{1}\left\{G_{t-s}(x+h, y)-G_{t-s}(x, y)\right\} \psi(X(s, y)) \mathrm{d} y \mathrm{~d} s\right)\right\} .
\end{aligned}
$$

Because of Lemma A1.1 (a) and Corollary 4.2, $T_{11}$ is of order $h$ since $x \mapsto G_{t}\left(x, X_{0}\right)$ is Lipschitz continuous if $X_{0}$ is of class $C^{1}$. On the other hand, defining $U_{t, x}(h)=$ $\int_{0}^{t} \int_{0}^{1}\left\{G_{t-s}(x+h, y)-G_{t-s}(x, y)\right\} \psi(X(s, y)) \mathrm{d} y \mathrm{~d} s$, we clearly have

$$
\mathbb{E}\left|U_{t, x}(h)\right|^{p} \leqslant C_{p}\left(\int_{0}^{t} \int_{0}^{1}\left|G_{t-s}(x+h, y)-G_{t-s}(x, y)\right| \mathrm{d} y \mathrm{~d} s\right)^{p},
$$

and, applying Lemma A1.1 (b), for all $\gamma \in] \frac{3}{2} ; 3\left[\right.$, we get $\mathbb{E}\left|U_{t, x}(h)\right|^{p} \leqslant C_{p, \gamma} h^{(3-\gamma) p / \gamma}$; for $\gamma$ close to $\frac{3}{2}$, this gives a bound of order $h^{1-\epsilon}$. (Note that the method employed in Section 4.2 is no longer valid since, in Lemma A1.1 (b), $\frac{3}{2}$ is not in the interval; this accounts for the difference between time regularity and space regularity.)

Using the same computations as those of Section 4.2, we get $\mathbb{E}\left\|D^{M} U_{t, x}(h)\right\|^{p} \leqslant$ $C_{M p} h^{(3-\gamma) p / \gamma}$. Therefore, Corollary 4.1 can be applied, which yields the required bound for $T_{13}$.

We now deal with $T_{12}$; owing to the duality formula between $\delta$ and $D$, we have as before

$$
T_{12}=\mathbb{E}\left(\int_{0}^{t} \int_{0}^{1} f^{\prime \prime}(X(t, x)) D_{s, y} X(t, x)\left\{G_{t-s}(x+h, y)-G_{t-s}(x, y)\right\} \varphi(X(s, y)) \mathrm{d} y \mathrm{~d} s\right) .
$$

Set $V_{t, x}(h)=\int_{0}^{t} \int_{0}^{1} D_{s, y} X(t, x)\left\{G_{t-s}(x+h, y)-G_{t-s}(x, y)\right\} \varphi(X(s, y)) \mathrm{d} y \mathrm{~d} s$. Using the same method as in Section 4.2, we easily get

$$
\mathbb{E}\left|V_{t, x}(h)\right|^{p} \leqslant C_{p, \tau} \mathbb{E}\left|\int_{0}^{t} \int_{0}^{1} G_{t-s}(x, y)\left\{G_{t-s}(x+h, y)-G_{t-s}(x, y)\right\} \mathrm{d} y \mathrm{~d} s\right|^{p},
$$

and similar estimates for the derivatives of $V_{t, x}(h)$. Hence one has to consider the regularity w.r.t. $h$ of

$$
\int_{0}^{t} \int_{0}^{1}\left|\left(G_{t-s}(x+h, y)-G_{t-s}(x, y)\right) G_{t-s}(x, y)\right| \mathrm{d} y \mathrm{~d} s .
$$

Unfortunately, as can be seen in the proof of the Lemma A1.3 of Appendix 1, one cannot find a suitable bound for (4.8). Therefore, using the Hölder inequality, we first bound integral (4.8) by a constant times

$$
\left(\int_{0}^{t} \int_{0}^{1}\left|\left\{G_{t-s}(x+h, y)-G_{t-s}(x, y)\right\} G_{t-s}(x, y)\right|^{\gamma} \mathrm{d} y \mathrm{~d} s\right)^{1 / \gamma},
$$

where $\gamma$ is a real value greater than 1 but close to 1 . Then, by means of inequality (A1.2) in 
Lemma A1.3 of Appendix 1, we obtain that this term is of order $h^{(3-2 \gamma) / \gamma}$. The end of the proof is then similar to that of estimate (4.1), and we finally obtain that, for all $\epsilon \in] 0,1[$, there exists $C_{\epsilon, \tau}$ such that

$$
|\mathbb{E}[f(X(t, x+h))-f(X(t, x))]| \leqslant C_{\tau}\left\|F_{f}\right\|_{\infty} h^{1-\epsilon} .
$$

\subsection{Proof of estimates (4.3) and (4.4)}

We first deal with (4.3). Using the integration-by-parts formula (P2) and the property (P1) of continuity of $\delta$, we have

$$
\mid \mathbb{E}\left[f ( X ( t , x ) ] | = | \mathbb { E } \left\{F_{f}\left(X(t, x) \delta\left(\frac{D X(t, x)}{\|D X(t, x)\|^{2}}\right)\right\} \mid \leqslant\left\|F_{f}\right\|_{\infty}\left\|\frac{D X(t, x)}{\|D X(t, x)\|^{2}}\right\|_{1,2, H} .\right.\right.
$$

Then Proposition 3.1 yields the result.

We now concentrate on (4.4). The quantity that we consider is the following:

$$
\mathbb{E}\left[\Delta_{h}^{2} f(X(t, \cdot))(x)\right]=\mathbb{E}[f(X(t, x+h))+f(X(t, x-h))-2 f(X(t, x))] .
$$

As previously, we use a Taylor expansion and write, for $\epsilon \in\{-1,1\}$,

$$
\begin{aligned}
f(X(t, x+\epsilon h))-f(X(t, x)=\{ & X(t, x+\epsilon h)-X(t, x)\} f^{\prime}(X(t, x)) \\
& +\{X(t, x+\epsilon h)-X(t, x)\}^{2} \int_{0}^{1}(1-v) f^{\prime \prime}\left(Y_{t, x}^{(\epsilon)}(h, v)\right) \mathrm{d} v,
\end{aligned}
$$

where $Y_{t, x}^{(\epsilon)}(h, v)=X(t, x)+v\{X(t, x+\epsilon h)-X(t, x)\}$. Therefore,

$$
\begin{aligned}
\mathbb{E}\left[\Delta_{h}^{2} f(X(t, \cdot))(x)\right]= & \mathbb{E}\left[f^{\prime}(X(t, x)) \Delta_{h}^{2} X(t, \cdot)(x)\right] \\
& +\mathbb{E}\left(\{X(t, x+h)-X(t, x)\}^{2} \int_{0}^{1}(1-v) f^{\prime \prime}\left(Y_{t, x}^{(1)}(h, v)\right) \mathrm{d} v\right) \\
& +\mathbb{E}\left((X\{t, x-h)-X(t, x)\}^{2} \int_{0}^{1}(1-v) f^{\prime \prime}\left(Y_{t, x}^{(-1)}(h, v)\right) \mathrm{d} v\right) \\
& =T_{1}+T_{2}^{(1)}+T_{2}^{(-1)} .
\end{aligned}
$$

$T_{2}^{(1)}$ and $T_{2}^{(-1)}$ are easily handled, using Lemma 4.3 and Corollary 4.1 .

We now turn to $T_{1}$. Using the evolution equation (1.1), we write $T_{1}=T_{11}+T_{12}+T_{13}$, with 


$$
\begin{aligned}
& T_{11}=\mathbb{E}\left[f^{\prime}(X(t, x)) \int_{0}^{1} \Delta_{h}^{2} G_{t}(\cdot, y)(x) X_{0}(y) \mathrm{d} y\right], \\
& T_{12}=\mathbb{E}\left[f^{\prime}(X(t, x)) \int_{0}^{t} \int_{0}^{1} \Delta_{h}^{2} G_{t-s}(\cdot, y)(x) \psi(X(s, y)) \mathrm{d} y \mathrm{~d} s\right], \\
& T_{13}=\mathbb{E}\left[f^{\prime}(X(t, x)) \int_{0}^{t} \int_{0}^{1} \Delta_{h}^{2} G_{t-s}(\cdot, y)(x) \varphi(X(s, y)) W(\mathrm{~d} y, \mathrm{~d} s)\right] .
\end{aligned}
$$

Now, we have

$$
\left|\Delta_{h}^{2} \phi\right| \leqslant\left|\Delta_{h} \phi\right|+\left|\Delta_{-h} \phi\right| .
$$

Then, since $X_{0}$ is of class $C^{1}$, Lemma A1.1 (a) of Appendix 1 and Corollary 4.1 clearly yield $\left|T_{11}\right| \leqslant C h\left\|F_{f}\right\|_{\infty}$.

To deal with $T_{12}$, we study $U_{t, x, h}=\int_{0}^{t} \int_{0}^{1} \Delta_{h}^{2} G_{t-s}(\cdot, y)(x) \psi(X(s, y)) \mathrm{d} y \mathrm{~d} s$. Using the fact that $\psi$ is bounded, we have, for all $\gamma>1$,

$$
\mathbb{E}\left|U_{t, x ; h}\right|^{p} \leqslant C_{p}\left(\int_{0}^{t} \int_{0}^{1}\left|\Delta_{h}^{2} G_{t-s}(\cdot, y)(x)\right| \mathrm{d} y \mathrm{~d} s\right)^{p} \leqslant C_{p, \gamma}\left(\int_{0}^{t} \int_{0}^{1}\left|\Delta_{h}^{2} G_{t-s}(\cdot, y)(x)\right|^{\gamma} \mathrm{d} y \mathrm{~d} s\right)^{p / \gamma} .
$$

At this stage of the proof, we see that we need a finer estimate than that provided by inequality (4.12) and Lemma A1.1 (b). Indeed, in order to obtain the required estimate of order $h$, we would like to choose $\gamma=\frac{3}{2}$, and that is not possible in Lemma A1.1 (b). We then need a new esitmate which would be more adapted to the specific quantity $\Delta_{h}^{2} G$, estimate which is provided by Lemma A1.4 (a) of Appendix 1 with $\gamma=\frac{3}{2}$, and that yields $\mathbb{E}\left|U_{t, x, h}\right|^{p} \leqslant C_{p} h^{p}$. As for the derivatives, they are treated the usual way via Proposition 3.1 .

We now deal with $T_{13}$; as before, the duality between $\delta$ and the derivative operator yields

$$
T_{13}=\mathbb{E}\left(f^{\prime \prime}(X(t, x)) \int_{0}^{t} \int_{0}^{1} \Delta_{h}^{2} G_{t-s}(\cdot, y)(x) D_{s, y} X(t, x) \varphi(X(s, y)) \mathrm{d} y \mathrm{~d} s\right) .
$$

Set $K_{t, x}(h)=\int_{0}^{t} \int_{0}^{1} \Delta_{h}^{2} G_{t-s}(\cdot, y)(x) D_{s, y} X(t, x) \varphi(X(s, y)) \mathrm{d} y \mathrm{~d} s$. Using once more the method of Section 4.2 , one easily gets

$$
\mathbb{E}\left|K_{t, x}(h)\right|^{p} \leqslant C_{p, \tau} \mathbb{E}\left|\int_{0}^{t} \int_{0}^{1} G_{t-s}(x, y) \Delta_{h}^{2} G_{t-s}(\cdot, y)(x) \mathrm{d} y \mathrm{~d} s\right|^{p},
$$

and similar estimates for the derivatives of $K_{t, x}(h)$. Hence Lemma A1.4 of Appendix 1 and Corollary 4.1 finally yield

$$
\left|T_{12}\right| \leqslant C_{\tau} h\left\|F_{f}\right\|_{\infty}
$$

which completes the proof. 


\section{Appendix 1}

We start this appendix with some fundamental estimates for the Green kernels with the Neumann and the Dirichlet boundary conditions, kernels which are indifferently denoted by $G$. These estimates correspond to Lemmas A.2 and B.1 of Bally et al. (1995).

\section{Lemma A.1.}

(a) Let $h$ be a $2 \beta$-Lipschitz function, with $\beta>0$. Then, for all $x, x^{\prime}, t, t^{\prime}$,

$$
\left|\int_{0}^{1} G_{t^{\prime}}\left(x^{\prime}, y\right) h(y) \mathrm{d} y-\int_{0}^{1} G_{t}(x, y) h(y) \mathrm{d} y\right| \leqslant\|h\|_{\operatorname{Lip} 2 \beta}\left(\left|t^{\prime}-t\right|^{\beta}+\left|x^{\prime}-x\right|^{2 \beta}\right),
$$

where $\|h\|_{\operatorname{Lip} 2 \beta}=\sup _{x \neq y}\left(|h(y)-h(x)| /|y-x|^{2 \beta}\right)$.

(b) For $\beta \in] \frac{3}{2}, 3[$, there exists $C>0$ such that, for all $x, y$, we have

$$
\int_{0}^{t} \int_{0}^{1}\left|G_{t-r}(x, z)-G_{t-r}(y, z)\right|^{\beta} \mathrm{d} z \mathrm{~d} r \leqslant C|x-y|^{3-\beta} .
$$

(c) For all $\beta \in] 1,3[$ there exists $C>0$ such that, for all $(s, t)$ with $s \leqslant t$ and for all $x$,

$$
\begin{aligned}
\int_{s}^{t} \int_{0}^{1}\left|G_{t-r}(x, y)\right|^{\beta} \mathrm{d} y \mathrm{~d} r & \leqslant C|t-s|^{(3-\beta) / 2}, \int_{0}^{s} \int_{0}^{1}\left|G_{t-r}(x, y)-G_{s-r}(x, y)\right|^{\beta} \mathrm{d} y \mathrm{~d} r \\
& \leqslant C|t-s|^{(3-\beta) / 2}
\end{aligned}
$$

The following result is a mere consequence of Lemma 3.3 of Bally et al. (1994).

Lemma A1.2. Let $\eta \in] 0,1[$. There exists a constant $C$ such that, for all $x$ and all $t>\eta$,

$$
\int_{t-\eta}^{t} \int_{0}^{1} G_{t-s}^{2}(x, y) \mathrm{d} y \mathrm{~d} s \geqslant C \eta^{1 / 2},
$$

where $G$ denotes here the Green kernel corresponding to the Neumann boundary conditions.

Remark. A similar result holds for the Green kernel corresponding to the Dirichlet boundary conditions: it suffices to take $x$ in an interval $[\alpha, 1-\alpha]$, with $\alpha>0$.

We now state and prove technical lemmas which are crucial for our estimations.

Lemma A1.3. There exist constants $C$ and $C_{\gamma}$ such that, for all $t>0, x \in[0,1], h>0$ with $t+h \leqslant T$ and $x+h \leqslant 1$,

$$
\int_{0}^{t} \int_{0}^{1} G_{t-s}(x, y)\left|G_{t+h-s}(x, y)-G_{t-s}(x, y)\right| \mathrm{d} y \mathrm{~d} s \leqslant C h^{1 / 2}
$$

and 


$$
\int_{0}^{t} \int_{0}^{1}\left\{G_{t-s}(x, y)\left|G_{t-s}(x+h, y)-G_{t-s}(x, y)\right|\right\}^{\gamma} \mathrm{d} y \mathrm{~d} s \leqslant C_{\gamma} h^{3-2 \gamma},
$$

where $\gamma \in] 1, \frac{3}{2}[$.

Proof. Since $G_{t}(x, y)=\left\{1 /(2 \pi t)^{1 / 2}\right\} \exp \left\{-(x-y)^{2} / 4 t\right\}+\bar{G}_{t}(x, y), \quad$ where $\quad(t, x, y) \mapsto$ $\bar{G}_{t}(x, y)$ is in $C^{\infty}\left([0, T] \times \mathbb{R}^{2}\right)$, the behaviour of the integral in (A1.1) is determined by that of

$$
J(t, x, h)=\int_{0}^{t} \int_{-\infty}^{+\infty} g(t-s ; x-y)|g(t+h-s ; x-y)-g(t-s ; x-y)| \mathrm{d} y \mathrm{~d} s,
$$

where $g(t ; x)=\left(1 / t^{1 / 2}\right) \exp \left(-x^{2} / 4 t\right)$. Then, setting $z=(x-y) / h^{1 / 2}, \quad v=(t-s) / h$, in (A1.3), one easily sees that (A1.1) holds provided that

$$
J:=\int_{0}^{+\infty} \int_{-\infty}^{+\infty} g(v ; z)|g(v+1 ; z)-g(v ; z)| \mathrm{d} z \mathrm{~d} v<\infty .
$$

Similarly, if $z=(x-y) / h, v=(t-s) / h^{2}$, (A1.2) holds provided that

$$
J_{\gamma}:=\int_{0}^{+\infty} \int_{-\infty}^{+\infty}\{g(v ; z)|g(v ; z+1)-g(v ; z)|\}^{\gamma} \mathrm{d} z \mathrm{~d} v<\infty .
$$

The following standard identities will be used repeatedly:

$$
\int_{\mathbb{R}}|y|^{r} \exp \left(-\frac{y^{2}}{4 t}\right) \mathrm{d} y=C_{r} t^{(r+1) / 2},
$$

where $C_{r}$ is a constant that does not depend on $t$.

Now, for an arbitrary $f \in C^{1}(\mathbb{R})$, the following simple identity holds:

$$
f(x+1)-f(x)=\int_{0}^{1} f^{\prime}(u+x) \mathrm{d} u .
$$

Then, applying (A1.5) to $f(v)=g(v ; z)$, using the fact that there exist some constants $c, C$ such that, for all $t, x,|(\partial g / \partial t)(t ; x)| \leqslant(C / t) g(c t ; x)$, the Hölder inequality on [0, 1] yields

$$
\int_{\mathbb{R}} g(v ; z)|g(v+1 ; z)-g(v ; z)| \mathrm{d} z \leqslant \frac{C}{v^{3 / 2}},
$$

and hence there is integrability near infinity. As for the behaviour near zero, one has

$$
g(v ; z)|g(v+1 ; z)-g(v ; z)| \leqslant g^{2}(v ; z)+g(v ; z) g(v+1 ; z),
$$

and hence the integral w.r.t. $z$ of the right-hand side is bounded by a term of order $v^{-1 / 2}$ plus another which is defined for $v=0$. Thus $J$ is convergent.

As for $J_{\gamma}$, using (A1.5) and the Hölder inequality on $[0,1]$, one gets

$$
\int_{\mathbb{R}} g(v ; z)^{\gamma}|g(v ; z+1)-g(v ; z)|^{\gamma} \mathrm{d} z \leqslant C_{\gamma} \int_{\mathbb{R}} \int_{0}^{1} \frac{|u+z|^{\gamma}}{v^{2 \gamma}} \exp \left(-\gamma \frac{z^{2}+(z+u)^{2}}{4 v}\right) \mathrm{d} u \mathrm{~d} z .
$$


However, $\left\{(z+u)^{2}+z^{2}\right\} / 4 t=(1 / 2 t)\left\{(z+u / 2)^{2}+u^{2} / 4\right\}$; hence, by a change of variables, bounding $u$ by 1 and using (A1.4), we get

$$
\int_{\mathbb{R}} g(v ; z)^{\gamma}|g(v ; z+1)-g(v ; z)|^{\gamma} \mathrm{d} z \leqslant C_{\gamma} \frac{1}{v^{2 \gamma}} v^{(\gamma+1) / 2}=v^{(1-3 \gamma) / 2} .
$$

Therefore, since $\gamma>1$, we have $(1-3 \gamma) / 2<-1$, and the integral is convergent near infinity. (Note that, for $\gamma=1$, it is not.) As for the convergence near zero, we simply use the same method as in (A1.6).

We finally prove estimates for the iterated differences of the Green kernel $G$.

\section{Lemma A1.4.}

(a) For all $\gamma \in] 1,3[$, there exists a constant $C$ such that, for all $x, t, h$,

$$
\int_{0}^{t} \int_{0}^{1}\left|\Delta_{h}^{2} G_{t-s}(\cdot, y)(x)\right|^{\gamma} \mathrm{d} y \mathrm{~d} s \leqslant C_{\gamma} h^{3-\gamma} .
$$

(b) There exists a constant $C$ such that, for all $x, t, h$,

$$
\int_{0}^{t} \int_{0}^{1}\left|\Delta_{h}^{2} G_{t-s}(\cdot, y)(x)\right| \cdot G_{t-s}(x, y) \mathrm{d} y \mathrm{~d} s \leqslant C h .
$$

Proof. Using the same method as in Lemma A1.3, one easily sees that we only have to prove that

$$
K_{\gamma}:=\int_{0}^{+\infty} \int_{\mathbb{R}}\left|\Delta_{1}^{2} g(t ; \cdot)(x)\right|^{\gamma} \mathrm{d} x \mathrm{~d} t, \quad K:=\int_{0}^{+\infty} \int_{\mathbb{R}}\left|\Delta_{1}^{2} g(t ; \cdot)(y)\right| g(t ; y) \mathrm{d} y,
$$

are convergent. Now, instead of (A1.5), we use the following identity: if $f \in C^{2}(\mathbb{R})$, then

$$
\Delta_{1}^{2} f(x)=\int_{0}^{1} \int_{0}^{1} 2 v f^{\prime \prime}(x+(2 u-1) v) \mathrm{d} u \mathrm{~d} v .
$$

Set $K_{\gamma}(t)=\int_{\mathbb{R}}\left|\Delta_{1}^{2} g(t ; \cdot)(x)\right|^{\gamma} \mathrm{d} x$. Applying (A1.8) to $g(t ; \cdot)$ and using the Hölder inequality on $[0,1]^{2}$, one has

$$
K_{\gamma}(t) \leqslant \frac{C_{\gamma}}{t^{\gamma / 2}} \int_{\mathbb{R}} \int_{0}^{1} \int_{0}^{1} \frac{1}{t^{2 \gamma}}\left|\{x+(2 u-1) v\}^{2}-2 t\right|^{\gamma} \exp \left(-\frac{\gamma\{x+(2 u-1) v\}^{2}}{4 t}\right) \mathrm{d} u \mathrm{~d} v \mathrm{~d} x .
$$

Then, by a change of variables and (A1.4), we get $K_{\gamma}(t) \leqslant\left(C_{\gamma} / t^{5 \gamma / 2}\right) t^{(2 \gamma+1) / 2}=C_{\gamma} / t^{(3 \gamma-1) / 2}$; since $\gamma>1$, we have $(3 \gamma-1) / 2>1$, which gives the convergence near $+\infty$.

As for the convergence near zero, we simply use (4.12), which leads us to consider the same integral as in Lemma A1.1 (b), and hence to impose the condition $\gamma<3$.

As for (b), setting $K(t)=\int_{\mathbb{R}}\left|\Delta_{1}^{2} g(t ; \cdot)(y)\right| g(t ; y) \mathrm{d} y$, using (A1.8) and the Hölder inequality on $[0,1]^{2}$, we have

$$
K(t) \leqslant \frac{C}{t^{3}} \int_{\mathbb{R}} \int_{0}^{1} \int_{0}^{1} v\left|\{x+(2 u-1) v\}^{2}-2 t\right| \exp \left(-\frac{\left[\{x+(2 u-1) v\}^{2}+x^{2}\right]}{4 t}\right) \mathrm{d} u \mathrm{~d} v \mathrm{~d} x .
$$


Then, using the same method as in (A1.7), we get $K(t) \leqslant\left(C / t^{3}\right) t^{3 / 2}=C / t^{3 / 2}$, and hence the convergence is near infinity. As for the convergence near zero, we simply proceed as in (a).

\section{Acknowledgements}

The author wishes to thank Professor Vlad Bally, Professor Sylvie Méléard, Professor Annie Millet, Professor Etienne Pardoux and Professor Marta Sanz-Solé for their valuable remarks and corrections, as well as the anonymous referees.

\section{References}

Bally, V., Gyongy, I. and Pardoux, E. (1994) White-noise driven parabolic SPDEs with measurable drift. J. Funct. Anal., 120, 484-510.

Bally, V., Millet, A. and Sanz-Solé, M. (1995) Approximation and support theorem in Hölder norm for parabolic SPDE's. Ann. Probab., 23, 178-222.

Bally, V. and Pardoux E. (1998) Malliavin calculus for white-noise driven parabolic SPDEs. Potential Analysis, 9, pp 27-64.

Bergh, J. and Löfström, J. (1986) Interpolation Spaces: an Introduction. Berlin: Springer-Verlag.

Friedman, A. (1964) Partial Differential Equations of Parabolic Type. Englewood Cliffs, NJ: PrenticeHall.

Nualart, D. (1995) Malliavin Calculus and Related Fields. Berlin: Springer-Verlag.

Nualart, D. and Sanz, M. (1985) Malliavin calculus for two-parameter Wiener functionals. $Z$. Wahrscheinlichkeitstheorie Verw. Geb., 70, 573-590.

Walsh, J.B. (1986) An introduction to stochastic partial differential equations. Ecole d'été de Probabilités de Saint-Flour, pp. 266-437, Lecture Notes Math. 1180. Berlin: Springer-Verlag.

Received October 1995 and revised September 1997 